\title{
Feasibility of Dosimetry-Based High-Dose 131I-Meta-Iodobenzylguanidine with Topotecan as a Radiosensitizer in Children with Metastatic Neuroblastoma
}

\author{
Mark N. Gaze, ${ }^{1}$ Yen-Ch'ing Chang, ${ }^{1}$ Glenn D. Flux, ${ }^{2}$ Rob J. Mairs, ${ }^{3}$ Frank H. Saran, ${ }^{2}$ and \\ Simon T. Meller ${ }^{2}$ \\ ${ }^{1}$ Meyerstein Institute of Oncology, The Middlesex Hospital, London, United Kingdom \\ ${ }^{2}$ The Royal Marsden Hospital, London, United Kingdom \\ ${ }^{3}$ The University of Glasgow, Glasgow, United Kingdom
}

\begin{abstract}
Introduction: ${ }^{131}$ I-meta iodobenzylguanidine $\left({ }^{131} I-m I B G\right)$ therapy is established palliation for relapsed neuroblastoma. The topoisomerase-1 inhibitor, topotecan, has direct activity against neuroblastoma and acts as a radiation sensitiser. These 2 treatments are synergistic in laboratory studies. Theoretically, the benefit of ${ }^{131}$ I-mIBG treatment could be enhanced by dose escalation and combination with topotecan. Haematological support would be necessary to overcome the myelosuppression, which is the dose-limiting toxicity. Aims: Firstly, one aim of this study was to establish whether in vivo dosimetry could be used to guide the delivery of a precise total whole-body radiation-absorbed dose of 4 Gy accurately from $2{ }^{131} I-m I B G$ treatments. Secondly, the other aim of this study was to determine whether it is feasible to combine this treatment with the topotecan in children with metastatic neuroblastoma. Material and Methods: An activity of ${ }^{131} \mathrm{I}-\mathrm{mIBG}(12 \mathrm{mCi} / \mathrm{kg}, 444 \mathrm{MBq} / \mathrm{kg})$, estimated to give a whole-body absorbed-radiation dose of approximately 2 Gy, was administered on day 1, with topotecan $0.7 \mathrm{mg} / \mathrm{m}^{2}$ administered daily from days $1-5$. In vivo dosimetry was used to calculate a 2 nd activity of ${ }^{131} I-m I B G$, to be given on day 15 which would give a total whole-body dose of 4 Gy. A further 5 doses of topotecan were given from days 15-19. The myeloablative effect of this regimen was circumvented by peripheral blood stem cell or bone marrow support. Results: Eight children with relapsed stage IV neuroblastoma were treated. The treatment was delivered according to protocol in all patients. There were no unanticipated side-effects. Satisfactory haematological reconstituition occurred in all patients. The measured total whole-body radiation-absorbed dose ranged from 3.7 Gy to 4.7 Gy (mean, 4.2 Gy). Conclusions: In vivo dosimetry allows for a specified total whole-body radiation dose to be delivered accurately. This schedule of intensification of ${ }^{131} I-m I B G$ therapy by dose escalation and radiosensitization with topotecan with a haemopoietic autograft is safe and practicable. This approach should now be tested for efficacy in a phase II clinical trial.
\end{abstract}

Key words: neuroblastoma; meta-iodobenzylguanidine; topotecan; radionuclide therapy dosimetry

Address reprint requests to: Mark N. Gaze; University College London Hospitals National Health Service Foundation Trust, The Meyerstein Institute of Oncology, The Middlesex Hospital, Mortimer Street, London W1T 3AA, United Kingdom; Tel.: +44207380 9301; Fax: +4420 74360160

E-mail: mark.gaze@uclh.org

\section{INTRODUCTION}

\section{Neuroblastoma}

Neuroblastoma is a cancer which typically occurs in younger children. Most patients present with 
advanced disease where there are distant metastases in addition to the primary tumour. While the results of modern combined modality therapy are better than was previously the case, most patients with INSS stage $4^{1}$ neuroblastoma over the age of 1 year still die from their disease, and improved therapeutic strategies are necessary.

\section{${ }^{131}$ I-Meta-Iodobenzylguanidine}

The compound meta-iodobenzylguanidine (mIBG) is taken up by an active transport process into cells of the sympathetic nervous system origin by an active transport process ${ }^{2}$ involving the noradrenaline transporter molecule. Primary neuroblastoma tumors and secondary deposits usually accumulate mIBG. When appropriately radiolabeled, mIBG can be used for diagnostic scintigraphy or radionuclide therapy. Therapy of neuroblastoma with ${ }^{131} \mathrm{I}-\mathrm{mIBG}$ over the last 2 decades has largely been confined to the palliative treatment of patients with multiply relapsed disease. ${ }^{3}$ The principal side-effect encountered has been bone marrow suppression, especially thrombocytopenia. ${ }^{4}$ In general, low, nonmyeloablative activities have been administered, and only a few reports have attempted to quantify the whole-body radiation-absorbed $\operatorname{dose}^{5}$ (which may correlate with toxicity) or the tumor dose (which may correlate with response). ${ }^{6}$ There have been some reports of the use of higher activities of ${ }^{131} \mathrm{I}-\mathrm{mIBG}$ using peripheral blood stem cell or bone marrow support to circumvent toxicity. 7,8

\section{Whole-Body Dosimetry}

As there should be a relationship between the radiation-absorbed dose and the response, dose escalation offers one potential way to improve outcome. As higher whole-body radiation-absorbed doses are likely to be associated with greater toxicity, it becomes more important to accurately quantify the whole-body dose when greater activities are administered. Variations in pharmacokinetics, related to variations in the tumor burden and avidity of retention of ${ }^{131} \mathrm{I}-\mathrm{mIBG}$, mean that there is a poor correlation between the activity administered and the whole-body dose received between different patients. ${ }^{9}$ If, for an individual patient, a certain administered activity is given and the resulting whole-body dose is accurately measured, the activity required for a 2 nd administration aimed to give a particular desired whole-body dose can be estimated. The administration of high-dose ${ }^{131} \mathrm{I}-\mathrm{mIBG}$ therapy as 2 frac- tions, with the activity of the 2nd fraction prescribed in the light of the measured whole-body dose following the 1st fraction, should enable a desired total whole-body dose to be received more accurately than if all the radiopharmaceutical is delivered in a single administration.

\section{Topotecan}

Topotecan ${ }^{10}$ is a cytotoxic drug which has single-agent activity neuroblastoma, ${ }^{11}$ but, perhaps more importantly, it has also been shown to act as a radiosensitizer. ${ }^{12}$ Therefore, it may be possible to augment the potency of ${ }^{131} \mathrm{I}$-mIBG treatment by incorporating topotecan into the treatment schedule. Both in vitro (DNA disruption and clonogenic cell kill) and in vivo (murine xenograft regrowth delay) experimental models have been used to compare the effectiveness of 3 different ${ }^{131} \mathrm{I}-\mathrm{mIBG}$ and topotecan combination schedules with either ${ }^{131} \mathrm{I}$-mIBG or topotecan alone. These have shown that the combined treatment is synergistic, compared to single-modality therapy. The administration of topotecan together with or after ${ }^{131} \mathrm{I}-\mathrm{mIBG}$ produces greater effects than when it is administered prior to ${ }^{131} \mathrm{I}-\mathrm{mIBG} .{ }^{13}$

\section{Aims}

The first aim of this study was to establish whether in vivo dosimetry can be used to guide the delivery of a precise total whole-body radiation-absorbed dose of 4 Gy accurately from $2{ }^{131} \mathrm{I}-\mathrm{mIBG}$ treatments. The second aim of this study was to establish whether it is feasible to combine this treatment with the topotecan in children with metastatic neuroblastoma, using peripheral blood stem cell (or autologous bone marrow) support.

\section{PATIENTS AND METHODS}

\section{Patients}

Eight patients, 4 boys and 4 girls ranging between 5 and 12 years of age were treated with this schedule. All the children had heavily pretreated stage IV neuroblastoma, for which no potentially curative treatment was available. They were treated compassionately with palliative intent.

\section{Methods}

Following a decision that this treatment schedule (Fig. 1) was appropriate, and with fully informed parental consent, the 1 st procedure was an at- 


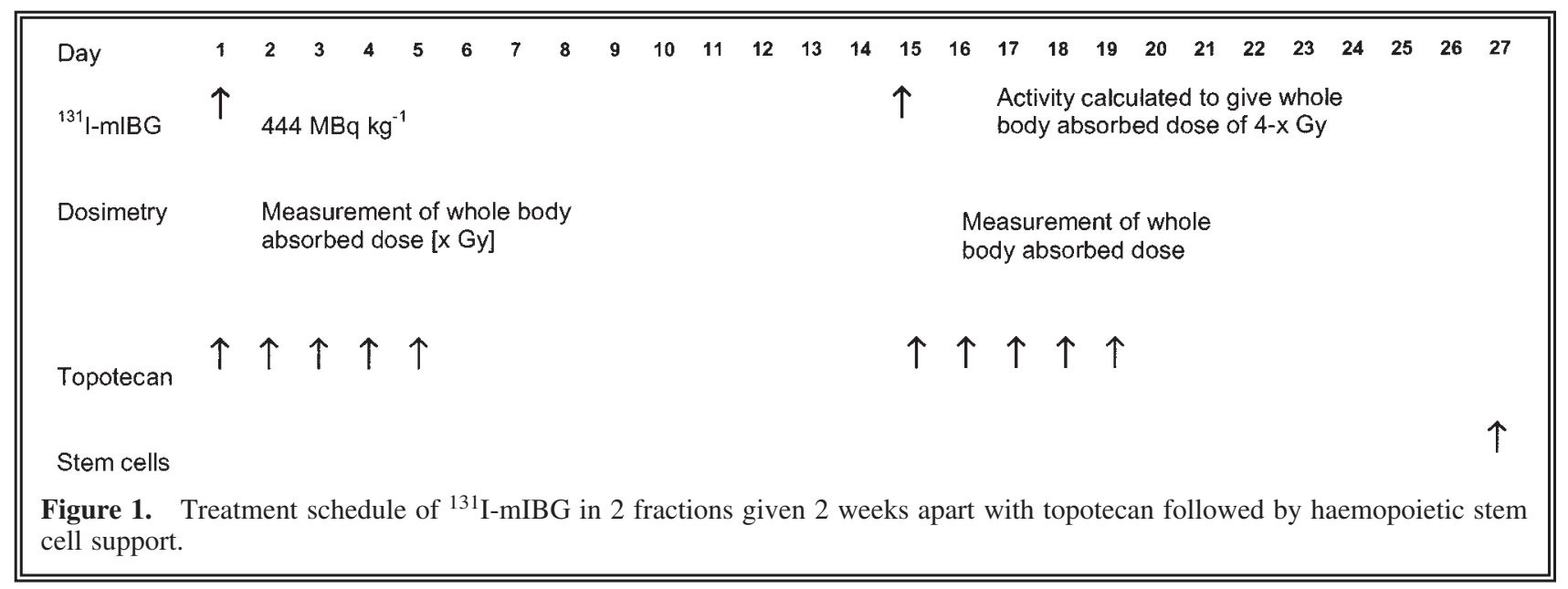

tempt to harvest peripheral blood stem cells. These were collected by apheresis after priming with cyclophosphamide and rh-GCSF, or on count recovery after cyclophosphamide, doxorubicin, and vincristine chemotherapy. When yields were less than $3 \times 10^{6} \mathrm{CD} 34+$ cells per kilogram (2 patients), a conventional bone marrow harvest was performed.

Patients were premedicated prior to treatment with granisetron $20 \mu \mathrm{g} / \mathrm{kg}^{-1}$ twice-daily, dexamethasone $100 \mu \mathrm{g} / \mathrm{kg}^{-1}$ twice-daily, potassium iodide (or equivalent) $250 \mathrm{mg} / \mathrm{m}^{-2} /$ day $^{-1}$ from day -1 to day 5 , and hydration was given with $0.9 \%$ saline $2 \mathrm{l} \mathrm{m}^{-2} / 24 \mathrm{hr}^{-1}$ from 4 hours before treatment for 24 hours to establish a diuresis.

Topotecan was reconstituted to a concentration between 25 and $50 \mu \mathrm{g} / \mathrm{mL}^{-1}$ in $5 \%$ dextrose. A dose of $0.7 \mathrm{mg} / \mathrm{m}^{-2}$ was infused over 30 minutes immediately prior to both ${ }^{131} \mathrm{I}$-mIBG administrations. Daily administrations were given for the subsequent 4 days.

The activity of ${ }^{131}$ I-mIBG for the 1 st administration on day 1 was calculated on the basis of the patient's weight. Prior to administration, the child was encouraged to empty his or her bladder. A precisely measured activity of about 444 $\mathrm{MBq} / \mathrm{kg}^{-1}\left(12 \mathrm{mCi} / \mathrm{kg}^{-1}\right)$ was infused over 30 minutes. The activity of ${ }^{131} \mathrm{I}-\mathrm{mIBG}$ for the 2 nd administration on day 15 was calculated on the basis of the measured whole-body radiation-absorbed dose following the 1 st administration. The aim was to give a total whole-body radiation-absorbed dose of $4 \mathrm{~Gy}$.

Whole-body radiation dose measurements were made using a ceiling-mounted energy-compensated geiger counter (Mini Instruments MC20 and MC70, Thermoelectron plc, Reading, UK). The patient was positioned supine on the bed, and care was taken to ensure that the patient positioning was the same for each measurement. Parents were trained to assist by taking measurements. A background measurement was taken prior to treatment. The initial measurement was taken immediately after ${ }^{131} \mathrm{I}-\mathrm{mIBG}$ infusion, and before the child emptied his or her bladder, at a precisely recorded time. Subsequently, measurements were taken approximately every $2-4$ hours at precisely recorded times. From these data, the whole-body radiation-absorbed dose was calculated. The activity of ${ }^{131} \mathrm{I}-\mathrm{mIBG}$ required for a 2nd administration, to give a total whole-body dose of $4 \mathrm{~Gy}$, was calculated, assuming that the pharmacokinetics remained the same. Stem cells were reinfused at, approximately, days 25-27, when the residual activity was less than $30 \mathrm{MBq}$. Supportive care was given as required, according to local protocols.

\section{RESULTS}

These 8 patients were treated at 2 hospitals. Two other hospitals were involved in stem cell harvest and supportive care. Treatment was given according to protocol in all cases. Peripheral blood stem cell rescue was used in 6 cases, and bone marrow was used in 2 cases. Supportive care with blood products and antibiotics was needed prior to haematological reconstitution. All patients satisfactorily re-engrafted, but count recovery was slower when marrow was used. No severe or unanticipated toxicity was seen.

Table 1 shows the administered activity of ${ }^{131} \mathrm{I}$-mIBG for both treatments for each patient, the measured whole-body radiation dose following each treatment, and the total for each 


\begin{tabular}{|c|c|c|c|c|}
\hline Patient & Treatment & $\begin{array}{l}\text { Administered } \\
\text { activity }(\mathrm{GBq})\end{array}$ & $\begin{array}{c}\text { Whole-body } \\
\text { dose (Gy) }\end{array}$ & $\begin{array}{l}\text { Total dose } \\
\text { (Gy) }\end{array}$ \\
\hline \multirow[t]{2}{*}{1} & 1 & 7.99 & 2.19 & \\
\hline & 2 & 6.85 & 2.16 & 4.35 \\
\hline \multirow[t]{2}{*}{2} & 1 & 9.25 & 2.25 & \\
\hline & 2 & 7.77 & 1.48 & 3.73 \\
\hline \multirow[t]{2}{*}{3} & 1 & 7.18 & 1.75 & \\
\hline & 2 & 9.25 & 2.90 & 4.65 \\
\hline \multirow[t]{2}{*}{4} & 1 & 7.03 & 1.70 & \\
\hline & 2 & 8.44 & 2.45 & 4.15 \\
\hline \multirow[t]{2}{*}{5} & 1 & 9.25 & 2.90 & \\
\hline & 2 & 3.59 & 0.93 & 3.83 \\
\hline \multirow[t]{2}{*}{6} & 1 & 13.3 & 2.44 & \\
\hline & 2 & 9.23 & 1.92 & 4.36 \\
\hline \multirow[t]{2}{*}{7} & 1 & 11.2 & 2.10 & \\
\hline & 2 & 9.73 & 2.20 & 4.30 \\
\hline \multirow[t]{2}{*}{8} & 1 & 6.87 & 2.20 & \\
\hline & 2 & 4.89 & 2.00 & 4.20 \\
\hline
\end{tabular}

patient. The mean whole-body dose following the 1st treatment in each patient, designed to be 2 Gy, was 2.19 Gy (range, 1.7-2.9 Gy). The mean variance from the intended dose of $2 \mathrm{~Gy}$ was $16 \%$ (range, $-15 \%-+45 \%$ ). The mean total whole-body dose from the 2 treatments, designed to be $4 \mathrm{~Gy}$, was 4.20 Gy (range, $3.73-4.65 \mathrm{~Gy})$. The mean variance from the intended total dose of 4 Gy was $7.7 \%$ (range, $-6.7 \%-+16.25 \%)$. These results are shown graphically in Figure 2.

\section{DISCUSSION}

These data show that delivery of this complex schedule of treatment is feasible in selected pa-

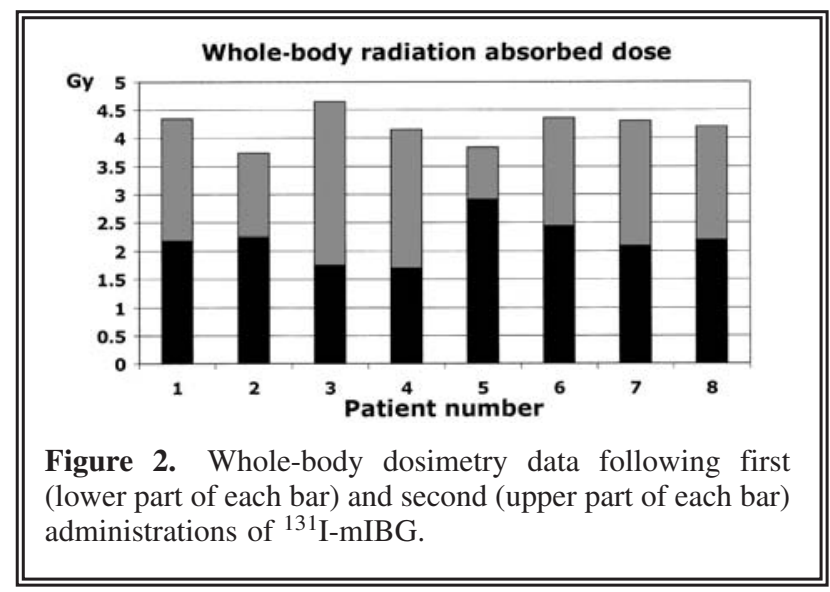

tients in well-equipped and resourced centers with an interest in paediatric radionuclide therapy. They show that it is feasible to give this treatment to patients referred from other centers, involving the referring hospital in shared supportive care remote from the treatment center.

The dosimetry data show that the use of in vivo whole-body radiation-absorbed dose measurements, following 1 treatment to guide the administered activity of a 2 nd fraction, enables a desired total whole-body dose to be given with significantly greater accuracy than is possible using an activity based only on the weight of the patient.

These findings are important for several reasons. Previously, an empirical activity of radionuclide was administered in clinical practice, which resulted in a highly variable whole-body absorbed radiation dose, and a highly variable tumor dose. The development of this system of standardized dosimetry has enabled a desired total whole-body radiation dose to be delivered much more accurately and precisely. This has, in turn, enabled dose escalation to be performed safely. As the received whole-body dose is now accurately predictable, toxicities can be anticipated reliably and dose escalation can be performed within the limits of normal tissue tolerance. Dose escalation, in this way, should result in higher and less variable tumor doses and better outcomes in terms of tumor control. Finally, 
the development of a standardized system of whole-body dosimetry, which can be replicated in a number of centers, will pave the way for multicenter clinical trials where dosimetry can be quality assured. In this way, there can be clinical confidence that the intended radionuclide doses have been delivered, and that the reported outcomes are the result of optimally delivered therapy and are, therefore, reliable.

\section{CONCLUSIONS}

As the aims of this study were related to feasibility and dosimetry, response assessment and survival were not reported. A larger multicenter phase II study is required to determine the response rate of this treatment package in a homogeneous cohort of patients treated earlier in the course of their illness.

\section{REFERENCES}

1. Brodeur GM, Seeger RC, Barrett A, et al. International criteria for diagnosis, staging and response to treatment in patients with neuroblastoma. J Clin Oncol 1988; 6:1874.

2. Mairs RJ, Gaze MN, Barrett A. The uptake and retention of meta-iodobenzyl guanidine by the neuroblastoma cell line NB1-G. Br J Cancer 1991;64:293.

3. Garaventa A, Guerra P, Arrighini A, et al. Treatment of advanced neuroblastoma with I-131 meta-iodobenzylguanidine. Cancer 1991;67:922.

4. Sisson JC, Hutchinson RJ, Shapiro B, et al. Toxicity from treatment of neuroblastoma with ${ }^{131}$ I-meta- iodobenzylguanidine. Eur J Nucl Med 1988;14: 337.

5. Du Bois SG, Messina J, Maris JM, et al. Haematologic toxicity of high-dose iodine-131-meta-iodobenzylguanidine therapy for advanced neuroblastoma. J Clin Oncol 2004;22:2452.

6. Flux GD, Guy MJ, Papavasileiou P, et al. Absorbed dose ratios for repeated therapy of neuroblastoma with I-131 mIBG. Cancer Biother Radiopharm 2003;18:81.

7. Gaze MN, Wheldon TE, O'Donoghue JA, et al. Multimodality megatherapy with $\left[{ }^{131} \mathrm{I}\right]$ meta-iodobenzylguanidine, high-dose melphalan and total-body irradiation with bone marrow rescue: Feasibility study of a new strategy for advanced neuroblastoma. Eur J Cancer 1995;31A:252.

8. Goldberg SS, De Santes K, Huberty JP, et al. Engraftment after myeloablative doses of ${ }^{131}$ I-meta-iodobenzylguanidine followed by autologous bone marrow transplantation for treatment of refractory neuroblastoma. Med Paediatr Oncol 1988;30:339.

9. Flux GD, Guy MJ, Beddows R, et al. Estimation and implications of random errors in whole-body dosimetry for targeted radionuclide therapy. Phys Med Biol 2002;7:3211.

10. Vassal G, Pondarré C, Capelli C, et al. DNA-topoisomerase I, a new target for the treatment of neuroblastoma. Eur J Cancer 1997;33:2011.

11. Nitschke R, Parkhurst J, Sullivan J, et al. Topotecan in pediatric patients with recurrent and progressive solid tumours: A Pediatric Oncology Group phase II study. J Pediatr Hematol/Oncol 1998;20:315.

12. Miyamoto S, Huang TT, Wuerzberger-Davis S, et al. Cellular and molecular responses to topoisomerase I poisons. Exploiting synergy for improved radiotherapy. Ann NY Acad Sci 2000;922:274.

13. Mairs RJ, McCluskey AG, Cosimo E, et al. [ $\left.{ }^{131} \mathrm{I}\right]$ metaiodobenzylguanidine and topotecan experimental combination treatment of tumors expressing the noradrenaline transporter. Pediatr Blood Cancer 2004;43:381. 